\title{
Soil greenhouse gas fluxes and net global warming potential from intensively cultivated vegetable fields in southwestern China
}

\author{
Z. J. Mu ${ }^{1,2.3^{*}}$, A.Y. Huang ${ }^{4}$, J.P. Ni ${ }^{1,2,3}$, J.Q Li ${ }^{5}$, Y. Y. Liu ${ }^{1}$, S. Shi ${ }^{1}$, D.T. Xie ${ }^{1,2,3}$, R. Hatano ${ }^{6}$
}

${ }^{1}$ Key Laboratory of Eco-environments in Three-Gorges Region of Ministry of Education, China. ${ }^{2}$ College of Resources and Environment, Southwest University, China. ${ }^{3}$ Engineering Research Center for Agricultural Non-point Source Pollution Control in Three-Gorges Region, China. ${ }^{4}$ College of Agronomy and Biotechnology, Southwest University, China. ${ }^{5}$ Meteorological Bureau of Beibei District, China. ${ }^{6}$ Research Faculty of Agriculture, Hokkaido University, Japan. "Correspondingauthor: muzj01@gmail. com

\begin{abstract}
Vegetable fields in China are characterized with intensive fertilization and cultivation, and their net effect on the global warming deserves attention. Greenhouse gas fluxes were thus measured, using a static closed chamber method, over approximately 18 months in two typical subtropical vegetable fields with different soil types and contrasting soil properties. Five consecutive crops were planted in one field and four in the other. Intensive fertilization consistently stimulated soil $\mathrm{N}_{2} \mathrm{O}$ emission, while imposed complicated impact on soil respiration with $\mathrm{CO}_{2}$ emission enhanced in one field and suppressed in the other field. The fertilizer-induced $\mathrm{N}_{2} \mathrm{O}$ emission factors (EFs) varied with individual crop phases and averaged 1.4 to $3.1 \%$ across the whole sampling period for different fields. The interaction of soil temperature and moisture could explain about $58 \%$ of the seasonal variation in the EFs. All the soils under different vegetable cropping systems were net sources of atmospheric radiative forcing and the net global warming potential over the entire study period ranged from 1,786 to $3,569 \mathrm{~g} \mathrm{CO}_{2}$ equivalence $\mathrm{m}^{-2}$ for fertilized soils with net $\mathrm{CO}_{2}$ emission contributing 53 to $67 \%$ and $\mathrm{N}_{2} \mathrm{O}$ emission occupying the remaining 33 to $47 \%$.
\end{abstract}

Keywords: Carbon dioxide, global warming potential, methane, nitrous oxide, vegetable fields 


\section{Introduction}

Rising atmospheric levels of the greenhouse gases carbon dioxide $\left(\mathrm{CO}_{2}\right)$, nitrous oxide $\left(\mathrm{N}_{2} \mathrm{O}\right)$, and methane $\left(\mathrm{CH}_{4}\right)$ have caused a constant increase in radiative forcing of the earth's atmosphere (IPCC, 2007). Global intensification of food and fiber production is an important factor influencing the budget of these greenhouse gases (GHGs). Agriculture and agriculture-related land-use change are estimated to contribute about one-third of the current total emission of these GHGs (Cole et al., 1997). Increasing world population imposes a challenge to increase agricultural production without increasing GHG emissions and environmental degradation (AdvientoBorbe et al., 2007).

As the country with the largest population, China gives great concern over its vegetable supply. The vegetable field has increased from 3 million ha in 1980 to 18.4 million ha in 2009 (Chinese Statistical Bureau, 2010), and the land management has been intensified greatly since the past few decades with large inputs of mineral fertilizer and other resources (Guo et al, 2010). The yearly cropping index of vegetable fields is generally over 2 to 3 in southern parts of China. Fertilizer N application rates during individual vegetable growing seasons ranged from 300 to $700 \mathrm{~kg} \mathrm{~N} \mathrm{ha}^{-1}$ and even more in some cases, while the national rate for all crops averaged $120 \mathrm{~kg} \mathrm{~N}^{-1}$ per crop (Zheng et al., 2004; He et al., 2009). Highly intensive cultivation of vegetable has the potential to change physical and chemical environment of the soil such as temperature and water content, and the availability of nutrients (Wang et al., 2008). Changes in these properties can control the rate and extent of microbial processes such as organic matter decomposition, denitrification and nitrification, which in turn control the carbon storage in soil and affect net exchange of the GHGs between the soil and the atmosphere. The increasing use of $\mathrm{N}$ fertilizers has induced intensive $\mathrm{N}_{2} \mathrm{O}$ emission from vegetable fields (He et al., 2009; Mei et al., 2011; Zhu et al., 2011). It is estimated that direct $\mathrm{N}_{2} \mathrm{O}$ emission from vegetable cultivation contributed about $20 \%$ of the national cropland emissions (Zheng et al., 2004). Moreover, soil organic carbon content was generally found to decline due to the application of mineral fertilizers nearly entirely replacing of traditional organic fertilizer or enhanced decomposition of organic matter with high-frequency cultivation practices (Wang et al., 2008; Tian et al., 2011). A complete understanding of vegetable farming's impact on climate change requires a full accounting of all contributors to the net global warming potential (GWP) of these systems (Robertson et al., 2000). However, there were so far limited field investigations making an integrated evaluation of the net effect of Chinese vegetable cultivation on global warming. Therefore, a monitoring campaign was conducted for two subtropical vegetable fields with contrasting soil properties to quantify the magnitude of $\mathrm{CO}_{2}, \mathrm{CH}_{4}$ and $\mathrm{N}_{2} \mathrm{O}$ fluxes and to estimate the net GWP of these soil-vegetable cropping systems.

\section{Materials and Methods}

\subsection{Field experiment}

The experiment was carried out from early March 2005 to middle September 2006 in two vegetable fields at Beibei District, Chongqing Municipality, China. One field (hereafter referred to as site CS) is located at Caoshang village $\left(29^{\circ} 47^{\prime} 05^{\prime \prime} \mathrm{N}, 106^{\circ} 26^{\prime} 31^{\prime \prime} \mathrm{E}, 585\right.$ $\mathrm{m}$ a.s.l) and the other (site $\mathrm{CJ}$ ) at Chengjiang village $\left(29^{\circ} 53^{\prime} 07^{\prime \prime} \mathrm{N}, 106^{\circ} 22^{\prime} 12^{\prime \prime}\right.$ E, $298 \mathrm{~m}$ a.s.l). The field at site CS has been cultivated with vegetables since the early 1980s after a long-term maize-wheat rotation, while the field at site $\mathrm{CJ}$ has been converted to vegetable cultivation in middle 1990s from rice paddies. The groundwater table in the field was not detectable for site CS but about $80 \mathrm{~cm}$ deep for site CJ. The soil at site CS was derived from Triassic limestone and classified as Typic Xanthi-Udic Ferralosol (CRGCST, 2001), with $\mathrm{pH}$ of 5.64 , organic $\mathrm{C}$ of 8.83 $\mathrm{g} \mathrm{kg}^{-1}$, total $\mathrm{N}$ of $1.50 \mathrm{~g} \mathrm{~kg}^{-1}$, clay content of $52.5 \%$ and 
The soil at site CJ was derived from Jurassic sandstone and shale, and classified as Typic PurliUdic Cambosol, with pH of 5.65, organic C of 14.1 $\mathrm{g} \mathrm{kg}^{-1}$, total $\mathrm{N}$ of $1.27 \mathrm{~g} \mathrm{~kg}^{-1}$, clay content of $19.7 \%$ and silt of $37.8 \%$.

Four treatments with three replications (plot size: $8 \mathrm{~m} \times 5 \mathrm{~m}$ ) were established at each field: bare soil that was not planted with crops and not fertilized (NPNF); soil was planted with crops but neither mineral fertilizer nor manure was applied (PNF); soil was planted with crops and mineral fertilizer $\mathrm{N}$ was applied (PCF); soil was planted with crops and both mineral fertilizer and manure were applied (PCFM). All of the field management including tillage and fertilization followed the local conventional practices. Five consecutive crops were planted at site CS and four at site CJ (except for the NPNF plots) as described in Table 1. The soil was hoed by hand to about $20 \mathrm{~cm}$ deep before the establishment of the next crop and harrowed regularly during crop growing seasons for soil loosening and weed removal. Fertilizer was side-dressed several times after the seedlings of vegetables were transplanted and survived. The total mineral $\mathrm{N}$ rate applied to the treatments PCF and PCFM over the entire study period was $846 \mathrm{~kg}$ $\mathrm{N} \mathrm{ha}^{-1}$ for site CS and $766 \mathrm{~kg} \mathrm{~N} \mathrm{ha}^{-1}$ for site CJ (Table 1). Manure was applied only to the PCFM plots in the form of dung and urine of human and pigs diluted with large amount of water. The total manure $\mathrm{N}$ rate was 149 and $99 \mathrm{~kg} \mathrm{~N} \mathrm{ha}^{-1}$ for sites CS and CJ, respectively (Table 2 and Table 3 ).

The climate of the study area is continental monsoon subtropical with humid winter. The mean annual air temperatures in 2005 and 2006 were 18.4 and $19.4{ }^{\circ} \mathrm{C}$, respectively; and the annual precipitations were 1223 and $965 \mathrm{~mm}$, respectively (Beibei District Meteorological Observatory 2006). A severe summer drought occurred in 2006 with the precipitation from July to August amounting to $31.7 \mathrm{~mm}$, which was only $7.1 \%$ of the precipitation during the same periods in 2005 .

\subsection{Gas flux measurements}

The fluxes of $\mathrm{CO}_{2}, \mathrm{~N}_{2} \mathrm{O}$ and $\mathrm{CH}_{4}$ from soils were measured using a closed chamber technique (Mu et al., 2006; Mu et al., 2008a). The sampling was conducted at a frequency of about three times per month between 09:00 and 12:00 hours on each sampling date. Gas samples from sites CS and CJ were generally taken in two consecutive days rather than on the same dates due to the inconvenient transportation. On each sampling event, one chamber was used at each of the replicated experimental plots, and headspace samples of $20 \mathrm{~mL}$ were withdrawn from each of the chambers at 0,10 and $20 \mathrm{~min}$, using a polypropylene syringe with a threeway stopcock, and transferred into a $10 \mathrm{~mL}$ vacuumed vial bottle. Gas samples were analyzed for $\mathrm{CO}_{2}, \mathrm{CH}_{4}$ and $\mathrm{N}_{2} \mathrm{O}$ concentrations using gas chromatographic systems equipped with TCD and FID (SHIMADZU GC-2014) and ${ }^{63} \mathrm{Ni}-\mathrm{ECD}$ (Varian CP-3800), respectively. Gas fluxes were calculated by a linear regression of gas concentrations in chamber against the time within $20 \mathrm{~min}$, and expressed as arithmetic means. All the determination coefficients $\left(\mathrm{R}^{2}\right)$ of the linear regression were greater than 0.94 in our study. The detection limit for $\mathrm{CO}_{2}, \mathrm{CH}_{4}$ and $\mathrm{N}_{2} \mathrm{O}$ fluxes was $\pm 2.2 \mathrm{mg} \mathrm{C} \mathrm{m}^{-2} \mathrm{~h}^{-1}, \pm 0.5 \mu \mathrm{g} \mathrm{C} \mathrm{m}^{-2} \mathrm{~h}^{-1}$ and $\pm 1.5 \mu \mathrm{g} \mathrm{N} \mathrm{m}^{-2}$ $\mathrm{h}^{-1}$, respectively. The cumulative fluxes were calculated assuming linear changes in gas emissions between two successive sampling dates and summing up over the individual crop growth periods. For convenience's sake, the short duration of fallow before the next crops was combined to the preceding crop seasons. Although we conducted at least one sampling within two to four days following most of the fertilization practices, there might remain some uncertainties in the estimates of cumulative GHG emissions due to the low sampling frequency that might have caught or missed some peaks of GHG (especially $\mathrm{N}_{2} \mathrm{O}$ ) fluxes. The total number of cumulative days was 562 for site CS (Table 2) and 523 for site CJ (Table 3). In this study, negative fluxes indicate the uptake of a given gas by the soil while positive fluxes indicate net emission from the soil. For $\mathrm{N}_{2} \mathrm{O}$, a fertilizer-induced emission factor $(\mathrm{EF})$ was also calculated as recommended by IPCC (2006). 
Table 1. Cultivation and fertilization management for intensive vegetable fields

\begin{tabular}{|c|c|c|c|c|c|c|c|}
\hline \multirow[t]{2}{*}{ Site } & \multirow[t]{2}{*}{ Crop phase } & \multirow{2}{*}{$\begin{array}{l}\text { Date of } \\
\text { transplanting }\end{array}$} & \multirow{2}{*}{ Date of harvest } & \multirow{2}{*}{$\begin{array}{l}\text { Date of } \\
\text { fertilization }\end{array}$} & \multicolumn{2}{|c|}{ Mineral fertilizer } & \multirow{2}{*}{$\begin{array}{c}\text { Urine with water } \\
\left(\mathrm{m}^{3} \mathrm{ha}^{-1}\right)\end{array}$} \\
\hline & & & & & type & rate $\left(\mathrm{kg} \mathrm{N} \mathrm{ha}^{-1}\right)$ & \\
\hline \multirow[t]{11}{*}{$\mathrm{CS}$} & 1-Cabbage & $2005-3-5$ & $2005-5-27$ & $2005-3-22$ & Urea & 35 & \\
\hline & & & & $2005-4-26$ & Urea & 185 & \\
\hline & 2-Cabbage & $2005-6-30$ & 2005-9-18 & 2005-8-16 & Urea & 96 & 35.7 \\
\hline & 3-Cabbage & $2005-9-25$ & $2006-2-3$ & 2005-10-30 & Urea & 39 & 28.6 \\
\hline & & & & 2005-11-8 & Urea & 96 & \\
\hline & & & & 2005-11-30 & Urea & 96 & 35.7 \\
\hline & 4-Cabbage & $2006-2-20$ & $2006-6-7$ & $2006-3-3$ & & & 35.7 \\
\hline & & & & $2006-3-7$ & Urea & 96 & \\
\hline & & & & $2006-3-25$ & Urea & 29 & \\
\hline & 5-Radish & $2006-6-12$ & No harvest* & $2006-6-22$ & Urea & 58 & 62.5 \\
\hline & & & & $2006-7-3$ & Urea & 116 & 62.5 \\
\hline \multirow[t]{7}{*}{ CJ } & 1-Maize & $2005-4-12$ & $2005-7-26$ & $2005-4-12$ & Urea & 126 & 28.6 \\
\hline & & & & $2005-5-7$ & Compound & 118 & \\
\hline & 2-Lettuce & $2005-8-31$ & $2005-10-27$ & 2005-9-10 & Urea & 84 & 9.1 \\
\hline & & & & $2005-9-25$ & $\mathrm{NH}_{4} \mathrm{HCO}_{3}$ & 62 & 42.9 \\
\hline & 3-Lettuce & $2005-11-7$ & $2006-4-20$ & $2006-3-7$ & Urea & 125 & \\
\hline & & & & 2006-3-18 & $\mathrm{NH}_{4} \mathrm{HCO}_{3}$ & 77 & 42.9 \\
\hline & 4-Pepper & 2006-5-11 & $2006-9-16$ & 2006-5-19 & Urea & 174 & 62.5 \\
\hline
\end{tabular}

*The seedling was dried to death due to severe drought.

Table 2. Greenhouse gas emission and net global warming potential (GWP) from soils under different treatments in the intensive vegetable field at site $\mathrm{CS}^{*}$

\begin{tabular}{|c|c|c|c|c|c|c|c|c|c|c|c|c|c|c|c|c|c|c|}
\hline \multirow[t]{3}{*}{ Crop phase } & \multirow[t]{3}{*}{ Treatment } & \multicolumn{2}{|c|}{$\mathrm{N}$ fertilization } & \multicolumn{2}{|c|}{ carbon input } & \multicolumn{3}{|c|}{ Mean flux } & \multirow{3}{*}{$\begin{array}{c}\text { Cumulative } \\
\text { days }\end{array}$} & \multicolumn{5}{|c|}{ Cumulative emission } & \multicolumn{4}{|c|}{ GWP } \\
\hline & & mineral & manure & manur & op residue & $\mathrm{CO}_{2}$ & $\mathrm{CH}_{4}$ & $\mathrm{~N}_{2} \mathrm{O}$ & & $\mathrm{CO}_{2}$ & net $\mathrm{CO}_{2}$ emission & $\mathrm{CH}_{4}$ & $\mathrm{~N}_{2} \mathrm{O}$ & $\mathrm{EF}$ & $\mathrm{CO}_{2}$ & $\mathrm{CH}_{4}$ & $\mathrm{~N}_{2} \mathrm{O}$ & Net GWP \\
\hline & & \multicolumn{2}{|c|}{$\mathrm{kgN} \mathrm{ha}^{-1}$} & \multicolumn{2}{|c|}{$\mathrm{kgC} \mathrm{ha}^{-1}$} & $\mathrm{mgC} \mathrm{m} \mathrm{m}^{-2} \mathrm{hr}^{-1}$ & $\mu \mathrm{gC} \mathrm{m}{ }^{-2} \mathrm{hr}^{-1}$ & $\mu \mathrm{gN} \mathrm{m}{ }^{-2} \mathrm{hr}^{-1}$ & & & $\mathrm{kgC} \mathrm{ha}^{-1}$ & & $\mathrm{kgN} \mathrm{ha}^{-1}$ & (\%) & & & $\mathrm{CO}_{2} \mathrm{~m}^{-2}$ & \\
\hline \multirow[t]{4}{*}{ 1-Cabbage } & NPNF & & & & & $39.9 \mathrm{~B}$ & $-14.4 \mathrm{~A}$ & $83.4 \mathrm{~B}$ & 117 & $1061 \mathrm{C}$ & 1061 & -0.5 & $2.1 \mathrm{~B}$ & & 389 & -2 & 98 & 486 \\
\hline & PNF & & & & 42 & $53.7 \mathrm{~B}$ & $-28.8 \mathrm{~A}$ & $32.2 \mathrm{~B}$ & 117 & $1460 \mathrm{BC}$ & 1019 & -0.8 & $0.8 \mathrm{~B}$ & & 374 & -3 & 37 & 408 \\
\hline & PCF & 220 & & & 63 & $66.7 \mathrm{AB}$ & $-39.6 \mathrm{~A}$ & $250 \mathrm{~A}$ & 117 & $1856 \mathrm{~B}$ & 998 & -1.0 & $6.8 \mathrm{~A}$ & 2.7 & 366 & -3 & 318 & 681 \\
\hline & PCFM & 220 & 24 & 84 & 67 & $90.5 \mathrm{~A}$ & $-20.5 \mathrm{~A}$ & 377 A & 117 & $2494 \mathrm{~A}$ & 910 & -0.6 & $10.4 \mathrm{~A}$ & 3.9 & 334 & -2 & 487 & 819 \\
\hline \multirow[t]{4}{*}{ 2-Cabbage } & NPNF & & & & & $46.8 \mathrm{~B}$ & $-24.8 \mathrm{~A}$ & $9.1 \mathrm{BC}$ & 87 & $964 \mathrm{~B}$ & 964 & -0.5 & $0.2 \mathrm{~B}$ & & 353 & -2 & 9 & 361 \\
\hline & PNF & & & & 37 & $61.8 \mathrm{AB}$ & $-1.9 \mathrm{~A}$ & $7.7 \mathrm{C}$ & 87 & $1346 \mathrm{~A}$ & 927 & -0.01 & $0.2 \mathrm{~B}$ & & 340 & 0 & 9 & 349 \\
\hline & PCF & 96 & & & 56 & $70.6 \mathrm{AB}$ & $-24.0 \mathrm{~A}$ & $44.3 \mathrm{AB}$ & 87 & $1564 \mathrm{~A}$ & 908 & -0.5 & $1.1 \mathrm{~A}$ & 0.9 & 333 & -2 & 52 & 383 \\
\hline & PCFM & 96 & 17 & 60 & 58 & $79.5 \mathrm{~A}$ & $-4.8 \mathrm{~A}$ & $72.3 \mathrm{~A}$ & 87 & $1629 \mathrm{~A}$ & 846 & -0.1 & $1.4 \mathrm{~A}$ & 1.1 & 310 & 0 & 66 & 375 \\
\hline \multirow{4}{*}{ 3-Cabbage } & NPNF & & & & & $12.4 \mathrm{C}$ & $-7.4 \mathrm{~A}$ & $4.3 \mathrm{~B}$ & 148 & $342 \mathrm{D}$ & 342 & -0.5 & $0.1 \mathrm{~B}$ & & 125 & -2 & 5 & 128 \\
\hline & PNF & & & & 40 & $22.2 \mathrm{BC}$ & $-21.1 \mathrm{~A}$ & $9.1 \mathrm{~B}$ & 148 & $667 \mathrm{C}$ & 302 & -0.6 & $0.2 \mathrm{~B}$ & & 111 & -2 & 9 & 118 \\
\hline & PCF & 231 & & & 60 & $36.8 \mathrm{AB}$ & $-2.5 \mathrm{~A}$ & $80.6 \mathrm{~A}$ & 148 & $1083 \mathrm{~B}$ & 282 & -0.5 & $2.8 \mathrm{~A}$ & 1.1 & 103 & -2 & 131 & 233 \\
\hline & PCFM & 231 & 31 & 102 & 62 & $42.1 \mathrm{~A}$ & $-9.0 \mathrm{~A}$ & $114 \mathrm{~A}$ & 148 & $1322 \mathrm{~A}$ & 178 & -0.4 & $3.6 \mathrm{~A}$ & 1.3 & 65 & -1 & 169 & 233 \\
\hline \multirow[t]{4}{*}{ 4-Cabbage } & NPNF & & & & & $18.2 \mathrm{~B}$ & $-12.9 \mathrm{AB}$ & $1.4 \mathrm{~B}$ & 112 & $500 \mathrm{C}$ & 500 & -0.4 & $0.03 \mathrm{~B}$ & & 183 & -1 & 1 & 183 \\
\hline & PNF & & & & 42 & $42.1 \mathrm{AB}$ & $-20.0 \mathrm{AB}$ & $2.7 \mathrm{~B}$ & 112 & $1200 \mathrm{~B}$ & 458 & -0.6 & $0.1 \mathrm{~B}$ & & 168 & -2 & 5 & 171 \\
\hline & PCF & 125 & & & 56 & $72.4 \mathrm{~A}$ & -26.3 B & $44.4 \mathrm{AB}$ & 112 & $2085 \mathrm{~A}$ & 444 & -0.7 & $1.1 \mathrm{AB}$ & 0.8 & 163 & -2 & 52 & 212 \\
\hline & PCFM & 125 & 17 & 58 & 62 & $70.5 \mathrm{~A}$ & $-9.4 \mathrm{~A}$ & $76.4 \mathrm{~A}$ & 112 & $2026 \mathrm{~A}$ & 380 & -0.3 & $2.2 \mathrm{~A}$ & 1.5 & 139 & -1 & 103 & 241 \\
\hline \multirow[t]{4}{*}{ 5-Radish } & NPNF & & & & & $23.0 \mathrm{~A}$ & $-5.6 \mathrm{~A}$ & $9.5 \mathrm{~A}$ & 98 & $640 \mathrm{~B}$ & 640 & -0.1 & $0.2 \mathrm{~B}$ & & 235 & 0 & 9 & 244 \\
\hline & PNF & & & & 28 & $49.1 \mathrm{~A}$ & $-10.2 \mathrm{~A}$ & $33.9 \mathrm{~A}$ & 98 & $1124 \mathrm{~A}$ & 612 & -0.2 & $0.6 \mathrm{~B}$ & & 224 & -1 & 28 & 252 \\
\hline & PCF & 174 & & & 35 & $48.2 \mathrm{~A}$ & $-17.9 \mathrm{~A}$ & $61.9 \mathrm{~A}$ & 98 & $1043 \mathrm{~A}$ & 605 & -0.3 & $1.2 \mathrm{~A}$ & 0.3 & 222 & -1 & 56 & 277 \\
\hline & PCFM & 174 & 60 & 216 & 35 & $51.3 \mathrm{~A}$ & $-8.8 \mathrm{~A}$ & $60.2 \mathrm{~A}$ & 98 & $1096 \mathrm{~A}$ & 389 & -0.3 & $1.1 \mathrm{AB}$ & 0.2 & 143 & -1 & 52 & 193 \\
\hline \multirow{4}{*}{$\begin{array}{l}\text { Whole study } \\
\text { period }\end{array}$} & NPNF & & & & & $27.5 \mathrm{C}$ & $-12.8 \mathrm{~A}$ & $22.6 \mathrm{~B}$ & 562 & $3507 \mathrm{C}$ & 3507 & -2.0 & $2.6 \mathrm{~B}$ & & 1286 & -7 & 122 & 1401 \\
\hline & PNF & & & & 189 & $44.9 \mathrm{~B}$ & $-17.3 \mathrm{~A}$ & $16.8 \mathrm{~B}$ & 562 & $5797 \mathrm{~B}$ & 3318 & -2.2 & $1.9 \mathrm{~B}$ & & 1217 & -7 & 89 & 1298 \\
\hline & PCF & 846 & & & 270 & $58.9 \mathrm{AB}$ & $-22.2 \mathrm{~A}$ & $100 \mathrm{~A}$ & 562 & $7631 \mathrm{~A}$ & 3237 & -3.0 & $13.0 \mathrm{~A}$ & 1.3 & 1187 & -10 & 609 & 1786 \\
\hline & PCFM & 846 & 149 & 520 & 284 & $66.9 \mathrm{~A}$ & $-10.8 \mathrm{~A}$ & $146 \mathrm{~A}$ & 562 & $8567 \mathrm{~A}$ & 2703 & -1.7 & $18.7 \mathrm{~A}$ & 1.7 & 991 & -6 & 876 & 1861 \\
\hline
\end{tabular}

*The data followed by the same letters within a column are not significantly different $(p>0.05)$ among different treatments in the same crop phases. 
That is, the difference between cumulative $\mathrm{N}_{2} \mathrm{O}$ emissions for each cropping phase in the fertilized plots PCF or PCFM and non-fertilized plot PNF was divided by the rate of nitrogen input through mineral fertilizer and manure. Simultaneously with each gas flux measurement, soil temperature at $5 \mathrm{~cm}$ was recorded next to each chamber by a handy digital thermometer (CT-220, Custom Corp., Japan). The soil temperature measurements from the replicated plots under the same treatments were averaged to produce a mean value for the treatment on each sampling date. Disturbed soil samples were collected at $0-10 \mathrm{~cm}$ depth from each treatment plot on each gas sampling date, and the soil samples from the same treatments were mixed and homogenized to one single sample to determine soil moisture by oven drying at $105{ }^{\circ} \mathrm{C}$ for $24 \mathrm{~h}$. Soil moisture was expressed as percentage of water-filled pore space (WFPS) using measured soil bulk density and assuming a particle density of 2.65 $\mathrm{g} \mathrm{cm}^{-3}$.

Table 3. Greenhouse gas emission and net global warming potential (GWP) from soils under different treatments in the intensive vegetable field at site $\mathrm{CJ}^{*}$

\begin{tabular}{|c|c|c|c|c|c|c|c|c|c|c|c|c|c|c|c|c|c|c|}
\hline \multirow[t]{3}{*}{ Crop phase } & \multirow[t]{3}{*}{ Treatment } & \multicolumn{2}{|c|}{$\mathrm{N}$ fertilization } & \multicolumn{2}{|c|}{ carbon input } & \multicolumn{3}{|c|}{ Mean flux } & \multirow{3}{*}{$\begin{array}{c}\text { Cumulative } \\
\text { days }\end{array}$} & \multicolumn{5}{|c|}{ Cumulative emission } & \multicolumn{4}{|c|}{ GWP } \\
\hline & & mineral & manure & manure & crop residue & $\mathrm{CO}_{2}$ & $\mathrm{CH}_{4}$ & $\mathrm{~N}_{2} \mathrm{O}$ & & $\mathrm{CO}_{2}$ & net $\mathrm{CO}_{2}$ emission & $\mathrm{CH}_{4}$ & $\mathrm{~N}_{2} \mathrm{O}$ & $\mathrm{EF}$ & $\mathrm{CO}_{2}$ & $\mathrm{CH}_{4}$ & $\mathrm{~N}_{2} \mathrm{O}$ & Net GWP \\
\hline & & \multicolumn{2}{|c|}{$\mathrm{kgN} \mathrm{ha}^{1}$} & \multicolumn{2}{|c|}{$\mathrm{kgC} \mathrm{ha}^{-1}$} & $\mathrm{mgC} \mathrm{m}^{-2} \mathrm{hr}^{-1}$ & $\mu \mathrm{gCm} \mathrm{m}^{-2} \mathrm{hr}^{-1}$ & $\mu g \mathrm{~N} \mathrm{~m}^{-2} \mathrm{hr}^{-1}$ & & & $\mathrm{kgC} \mathrm{ha}{ }^{-1}$ & & $\mathrm{kgN} \mathrm{ha}^{-1}$ & (\%) & & & $\mathrm{CO}_{2} \mathrm{~m}^{2}$ & \\
\hline \multirow[t]{4}{*}{ 1-Maize } & NPNF & & & & & $97.2 \mathrm{~B}$ & $5.1 \mathrm{~A}$ & $54.3 \mathrm{~B}$ & 141 & $3256 \mathrm{~B}$ & 3256 & 0.20 & $1.8 \mathrm{~B}$ & & 1194 & 1 & 84 & 1279 \\
\hline & PNF & & & & 460 & $143 \mathrm{~A}$ & $4.8 \mathrm{~A}$ & $52.9 \mathrm{~B}$ & 141 & $4457 \mathrm{~A}$ & 2796 & 0.10 & $1.4 \mathrm{~B}$ & & 1025 & 0 & 66 & 1091 \\
\hline & PCF & 244 & & & 525 & $122 \mathrm{AB}$ & $3.6 \mathrm{~A}$ & $212 \mathrm{~A}$ & 141 & $4135 \mathrm{~A}$ & 2731 & 0.05 & $7.2 \mathrm{~A}$ & 2.4 & 1001 & 0 & 337 & 1339 \\
\hline & PCFM & 244 & 15 & 52 & 570 & $121 \mathrm{AB}$ & $8.3 \mathrm{~A}$ & 297 & 141 & $4089 \mathrm{~A}$ & 2634 & 0.20 & $9.8 \mathrm{~A}$ & 3.2 & 966 & 1 & 459 & 1425 \\
\hline \multirow[t]{4}{*}{ 2-Lettuce } & NPNF & & & & & $35.6 \mathrm{~A}$ & $-6.5 \mathrm{~A}$ & $5.8 \mathrm{C}$ & 68 & $571 \mathrm{~B}$ & 571 & -0.10 & $0.1 \mathrm{D}$ & & 209 & 0 & 5 & 214 \\
\hline & PNF & & & & 154 & $73.3 \mathrm{~A}$ & $12.9 \mathrm{~A}$ & $73.2 \mathrm{BC}$ & 68 & $1243 \mathrm{~A}$ & 417 & 0.05 & $1.2 \mathrm{C}$ & & 153 & 0 & 56 & 209 \\
\hline & PCF & 146 & & & 220 & $70.0 \mathrm{~A}$ & $-10.7 \mathrm{~A}$ & $381 \mathrm{AB}$ & 68 & $1220 \mathrm{~A}$ & 351 & -0.10 & $5.6 \mathrm{~B}$ & 3.0 & 129 & 0 & 262 & 391 \\
\hline & PCFM & 146 & 28 & 90 & 227 & $74.4 \mathrm{~A}$ & $-1.4 \mathrm{~A}$ & $583 \mathrm{~A}$ & 68 & $1402 \mathrm{~A}$ & 254 & -0.10 & $8.5 \mathrm{~A}$ & 4.2 & 93 & 0 & 398 & 491 \\
\hline \multirow[t]{4}{*}{ 3-Lettuce } & NPNF & & & & & $31.6 \mathrm{~A}$ & $2.8 \mathrm{~A}$ & $15.3 \mathrm{~B}$ & 185 & $1322 \mathrm{~B}$ & 1322 & 0.10 & $1.0 \mathrm{AB}$ & & 485 & 0 & 47 & 532 \\
\hline & PNF & & & & 221 & $36.8 \mathrm{~A}$ & $-1.8 \mathrm{~A}$ & $7.2 \mathrm{~B}$ & 185 & $1533 \mathrm{AB}$ & 1101 & 0.02 & $0.3 \mathrm{~B}$ & & 404 & 0 & 14 & 418 \\
\hline & PCF & 202 & & & 247 & $41.5 \mathrm{~A}$ & $4.9 \mathrm{~A}$ & 45.4 & 185 & $1747 \mathrm{~A}$ & 1075 & 0.30 & $1.9 \mathrm{~A}$ & 0.8 & 394 & 1 & 89 & 484 \\
\hline & PCFM & 202 & 23 & 79 & 266 & $32.9 \mathrm{~A}$ & $0.4 \mathrm{~A}$ & 50. $7 \mathrm{~A}$ & 185 & $1378 \mathrm{~B}$ & 977 & -0.10 & $2.0 \mathrm{~A}$ & 0.8 & 358 & 0 & 94 & 452 \\
\hline \multirow[t]{4}{*}{ 4-Pepper } & NPNF & & & & & $72.6 \mathrm{~A}$ & $-5.3 \mathrm{~A}$ & $17.0 \mathrm{~A}$ & 129 & $2175 \mathrm{C}$ & 2175 & 0.02 & $0.4 \mathrm{~B}$ & & 798 & 0 & 19 & 816 \\
\hline & PNF & & & & 286 & $109 \mathrm{~A}$ & $-4.5 \mathrm{~A}$ & $86.0 \mathrm{~A}$ & 129 & $3524 \mathrm{~A}$ & 1889 & -0.02 & $2.7 \mathrm{~B}$ & & 693 & 0 & 126 & 819 \\
\hline & PCF & 174 & & & 358 & $87.7 \mathrm{~A}$ & $11.6 \mathrm{~A}$ & $372 \mathrm{~A}$ & 129 & $2767 \mathrm{BC}$ & 1817 & 0.10 & $11.6 \mathrm{~A}$ & 5.1 & 666 & 0 & 543 & 1210 \\
\hline & PCFM & 174 & 33 & 112 & 387 & $92.3 \mathrm{~A}$ & $4.5 \mathrm{~A}$ & $460 \mathrm{~A}$ & 129 & $2981 \mathrm{AB}$ & 1676 & 0.10 & $13.1 \mathrm{~A}$ & 5.0 & 615 & 0 & 613 & 1228 \\
\hline \multirow{4}{*}{$\begin{array}{l}\text { Whole study } \\
\text { period }\end{array}$} & NPNF & & & & & $62.5 \mathrm{~B}$ & $0.5 \mathrm{~A}$ & $26.8 \mathrm{~B}$ & 523 & $7324 \mathrm{C}$ & 7324 & 0.20 & $3.3 \mathrm{~B}$ & & 2685 & 1 & 155 & 2841 \\
\hline & PNF & & & & 1121 & $90.3 \mathrm{~A}$ & $1.2 \mathrm{~A}$ & $47.9 \mathrm{~B}$ & 523 & $10757 \mathrm{~A}$ & 6203 & 0.20 & $5.6 \mathrm{~B}$ & & 2274 & 1 & 262 & 2537 \\
\hline & PCF & 766 & & & 1350 & $81.1 \mathrm{AB}$ & $4.4 \mathrm{~A}$ & $214 \mathrm{~A}$ & 523 & 9868 B & 5974 & 0.30 & $26.3 \mathrm{~A}$ & 2.7 & 2190 & 1 & 1232 & 3423 \\
\hline & PCFM & 766 & 99 & 333 & 1450 & $79.6 \mathrm{AB}$ & $3.6 \mathrm{~A}$ & $287 \mathrm{~A}$ & 523 & $9852 \mathrm{~B}$ & 5541 & 0.10 & $33.4 \mathrm{~A}$ & 3.2 & 2032 & 0 & 1564 & 3596 \\
\hline
\end{tabular}

* The data followed by the same letters within a column are not significantly different $(p>0.05)$ among different treatments in the same crop phases.

\subsection{Calculation of net global warming potential (GWP)}

The net GWP from soils was calculated as sum of net emissions of $\mathrm{CO}_{2}, \mathrm{~N}_{2} \mathrm{O}$ and $\mathrm{CH}_{4}$ by converting each gas unit to $\mathrm{CO}_{2}$ equivalent at a 100 -yr time scale using a conversion factor of 1 for $\mathrm{CO}_{2}, 298$ for $\mathrm{N}_{2} \mathrm{O}$ and 25 for $\mathrm{CH}_{4}$ (IPCC, 2007). Following Mu et al. (2008b), net $\mathrm{CO}_{2}$ emission from a soil was estimated as the difference between carbon loss as $\mathrm{CO}_{2}$ through microbial decomposition of soil organic materials and carbon gains through crop residue retaining in soils at harvest and manure application. $\mathrm{CO}_{2}$ fluxes from the bare soil (NPNF) plots were produced primarily from microbial decomposition of soil organic matter (Hanson et al. 2000) and was taken as a surrogate of soil organic carbon decomposition rate. The $\mathrm{CO}_{2}$ fluxes from the treatments PCFM and PCF were generally not significantly different, therefore the decomposition loss of manure carbon applied to PCFM was not accounted for estimating net $\mathrm{CO}_{2}$ emission. Crop residue directly returning to the soils in the study region was mainly in the form of root biomass since the aboveground straw was conventionally taken home and used as bedding material for pigsty or firefuel. 
The root biomass of individual vegetable crops at harvest was obtained from ten plants for each treatment. A soil column with a size of $30 \mathrm{~cm}$ in diameter and depth was dug up around each of sample plants and visible root biomass was manually collected. The root samples were then dried at $80{ }^{\circ} \mathrm{C}$ and weighed, and ground for analysis of carbon content with a C-N analyzer (SUMIGRAPH NC-1000, Sumika Chemical Analysis Service, Japan).

\subsection{Statistical Analysis}

One-way ANOVA procedure with Fisher LSD test was used to compare the mean difference $(p<0.05)$ of a given variable between different treatments in the same crop phases. Relationships between variables were assessed using simple linear regression analysis. All the statistical analyses were conducted using the software SAS 9.3 for Windows.

\section{Results and Discussion}

\section{1. $\mathrm{CO}_{2}$ fluxes}

Soil $\mathrm{CO}_{2}$ fluxes generally followed changes in soil temperature with high fluxes occurring during the warm period from April to October and low fluxes during the cool period from November to March (Figure 1 and 2). Corresponding to frequent soil drying/rewetting events, large fluctuations in soil $\mathrm{CO}_{2}$ fluxes were also observed during the warm period of a year. In particular, the pattern of $\mathrm{CO}_{2}$ fluxes during the period from June to September in 2006 was consistent with the fluctuations in soil WFPS, with peak emissions observed in early September with soil WFPS increasing abruptly to around $60 \%$ from about $5 \%$ in August, probably due to the dubbed "Borken effect" (Rey et al., 2002). The linear regression analysis showed that soil WFPS could explain, on average, $69 \%$ of the variation in soil $\mathrm{CO}_{2}$ fluxes during this period for different treatments at sites CJ and $86 \%$ for site $\mathrm{CS}$. The soil $\mathrm{CO}_{2}$ fluxes in treatments with crops were contributed not only by microbial decomposition of organic matter but also by root respiration, and were therefore significantly greater than those in the treatment NPNF with bare soils $(p<0.05)$ for both sites on most of the study period. Across the whole study period, the average $\mathrm{CO}_{2}$ fluxes in different treatments ranged from 27.5 to $66.9 \mathrm{mg} \mathrm{C} \mathrm{m}^{-2} \mathrm{~h}^{-1}$ for site CS (Table 2), and from 62.5 to $81.1 \mathrm{mg} \mathrm{C} \mathrm{m}^{-2} \mathrm{~h}^{-1}$ for site CJ (Table 3).

The total soil $\mathrm{CO}_{2}$ emission in different treatments over the whole study period varied from 3,507 to $8,567 \mathrm{~kg} \mathrm{C} \mathrm{ha}^{-1}$ for site CS (Table 2) and from 7,324 to $10,757 \mathrm{~kg} \mathrm{C}^{-1}$ for site CJ (Table 3). The treatments PCF and PCFM showed 32 to $47 \%$ higher $\mathrm{CO}_{2}$ emission than PNF at site CS $(p<0.05)$, while the case was reverse at site $\mathrm{CJ}$ with PNF showing about $8 \%$ higher $\mathrm{CO}_{2}$ emission than PCF and PCFM, indicating that the application of mineral fertilizer had complicated effect on soil respiration. The stimulatory effect of $\mathrm{N}$ fertilization observed at site CS may result from high soil organic carbon decomposition with increasing microbial biomass and activity and/or more photosynthesized carbon released into soils as exudates or consumed through root respiration due to $\mathrm{N}$ addition (Liu et al., 2011). In contrast, $\mathrm{N}$ fertilization was also found to cause a reduction in fine root biomass of plants and microbial biomass (Jia et al., 2010), which may be responsible for the suppression of soil respiration by $\mathrm{N}$ addition observed at site CJ. The combined application of mineral fertilizer and manure at both sites did not influence $\mathrm{CO}_{2}$ emission greatly $(p>0.05)$ when compared with the treatment applied with mineral fertilizer only (PCF), probably due to the low application rate of manure carbon. Each of the treatments at site $\mathrm{CJ}$ tended to has a larger $\mathrm{CO}_{2}$ emission than the corresponding treatments at site $\mathrm{CS}$, and this difference could attribute mainly to the different decomposition rates of soil organic carbon between the two sites. The overall rate of soil organic carbon decomposition as indicated by the $\mathrm{CO}_{2}$ emission from the treatment NPNF was about 2 times lower at site CS than at site CJ. The lower decomposition rate at site CS may be due partly to the lower soil temperature and moisture and carbon substrate availability. The 
high content of soil silt plus clay at site CS may also absorb organic matter and protect it from being decomposed by microorganisms (Hassink et al., 1993; Mu et al., 2008b).

The balance of soil carbon loss as $\mathrm{CO}_{2}$ through organic matter decomposition and input as crop residue or manure determines whether a soil acts as a net sink or source of atmospheric $\mathrm{CO}_{2}$. The vegetables such as cabbage and lettuce have high harvest indices and the carbon retained in the soil at harvest is mainly in the form of root residue. The root residue was also the main form of carbon retained in soil for the grain or fruit crops such as maize and pepper in the study region since their aboveground straw was often taken home and used as firefuel or bedding material for pigsty. For all treatments at both sites, the amount of soil carbon gains from root residue and manure was not enough to offset the carbon loss through soil organic matter decomposition as indicated by the $\mathrm{CO}_{2}$ emission from bare soils. As a result, the soils showed a net $\mathrm{CO}_{2}$ emission to the atmosphere with the rate ranging from 2,703 to 3,507 $\mathrm{kg} \mathrm{C} \mathrm{ha}^{-1}$ for site CS (Table 2) and from 5,541 to $7,324 \mathrm{~kg} \mathrm{C}^{-1}$ for site CJ (Table 3). The net $\mathrm{CO}_{2}$ emission observed in this study was in the range reported for other upland cropping or fallow fields for which the net $\mathrm{CO}_{2}$ emission varying from 785 to 7,349 kg C ha-1 (Mu et al., 2008b).

\section{2. $\mathrm{CH}_{4}$ fluxes}

Different patterns of change in soil $\mathrm{CH}_{4}$ fluxes were observed for sites CS and CJ (Figure 1 and 2). The soils at site $\mathrm{CS}$ showed small $\mathrm{CH}_{4}$ uptake rates on most of the sampling dates and a few dates for which net emissions of $\mathrm{CH}_{4}$ were observed. The distinct episodes of $\mathrm{CH}_{4}$ emissions occurred following heavy precipitation during July to November 2005. Unlike site CS, the soils at site CJ acted alternatively as small sources or sinks of $\mathrm{CH}_{4}$. Across the entire study period, the mean fluxes of $\mathrm{CH}_{4}$ in different treatments varied from -22.3 to -10.8 $\mu \mathrm{g} \mathrm{C} \mathrm{m} \mathrm{m}^{-2} \mathrm{~h}^{-1}$ for site CS (Table 2), and from 0.5 to 4.4 $\mu \mathrm{g} \mathrm{C} \mathrm{m}^{-2} \mathrm{~h}^{-1}$ for site CJ (Table 3).
The soils in different treatments at site CS acted as a small sink of $\mathrm{CH}_{4}$ across the five vegetable growth phases and the overall uptake rates ranged from -3.0 to $-1.7 \mathrm{~kg} \mathrm{C} \mathrm{ha}^{-1}$ (Table 2). In contrast, the soils in different treatments at site CJ showed a small net emission during the maize growth phase and alternated to be a small sink or source during the other three vegetable growing phases (Table 3 ). Over the entire study period, the soils at site CJ acted as a small source of $\mathrm{CH}_{4}$ with emission rates ranging from 0.1 to $0.3 \mathrm{~kg} \mathrm{C} \mathrm{ha}^{-1}$. Neither fertilization nor soil covers (bare or crops) significantly influenced $\mathrm{CH}_{4}$ fluxes at both sites $(p>0.05)$.

\section{3. $\mathrm{N}_{2} \mathrm{O}$ fluxes}

In the treatments NPNF and PNF at both sites CS and $\mathrm{CJ}$, the seasonal pattern of $\mathrm{N}_{2} \mathrm{O}$ emission was characterized by low flux rates on most sampling dates and a few dates on which flux rates were higher than $50 \mu \mathrm{g} \mathrm{N} \mathrm{m}^{-2} \mathrm{~h}^{-1}$ (Figure 1 and 2). By contrast, the treatments PCF and PCFM showed a large seasonal variation in soil $\mathrm{N}_{2} \mathrm{O}$ emission with flux rates exceeding $50 \mu \mathrm{g} \mathrm{N} \mathrm{m}^{-2} \mathrm{~h}^{-1}$ on more than half of the sampling dates. At site CS, the highest emissions took place during the first cropping phase of cabbage (from April through June in 2005). Though a comparable rate of mineral $\mathrm{N}$ was applied in the treatments PCF and PCFM during the other four cropping phases (Table 1), the $\mathrm{N}_{2} \mathrm{O}$ fluxes from soils were relatively low and the mechanisms responsible for this seemed to be different (Figure 1). During the third cropping phase of cabbage (from late September 2005 to middle February 2006), soil moisture (around 55\% of WFPS) was close to that during the first cropping phase, but the mean soil temperature (about $13{ }^{\circ} \mathrm{C}$ ) was the lowest among all five cropping phases, which may limit microbial denitrification and nitrification activities (Maag and Vinther, 1999). ). On the other hand, the low soil moisture (mean values of WFPS ranging from 22 to $45 \%$ ) may be responsible for the low $\mathrm{N}_{2} \mathrm{O}$ fluxes during the remaining cropping phases (Linn and Doran, 1984). 


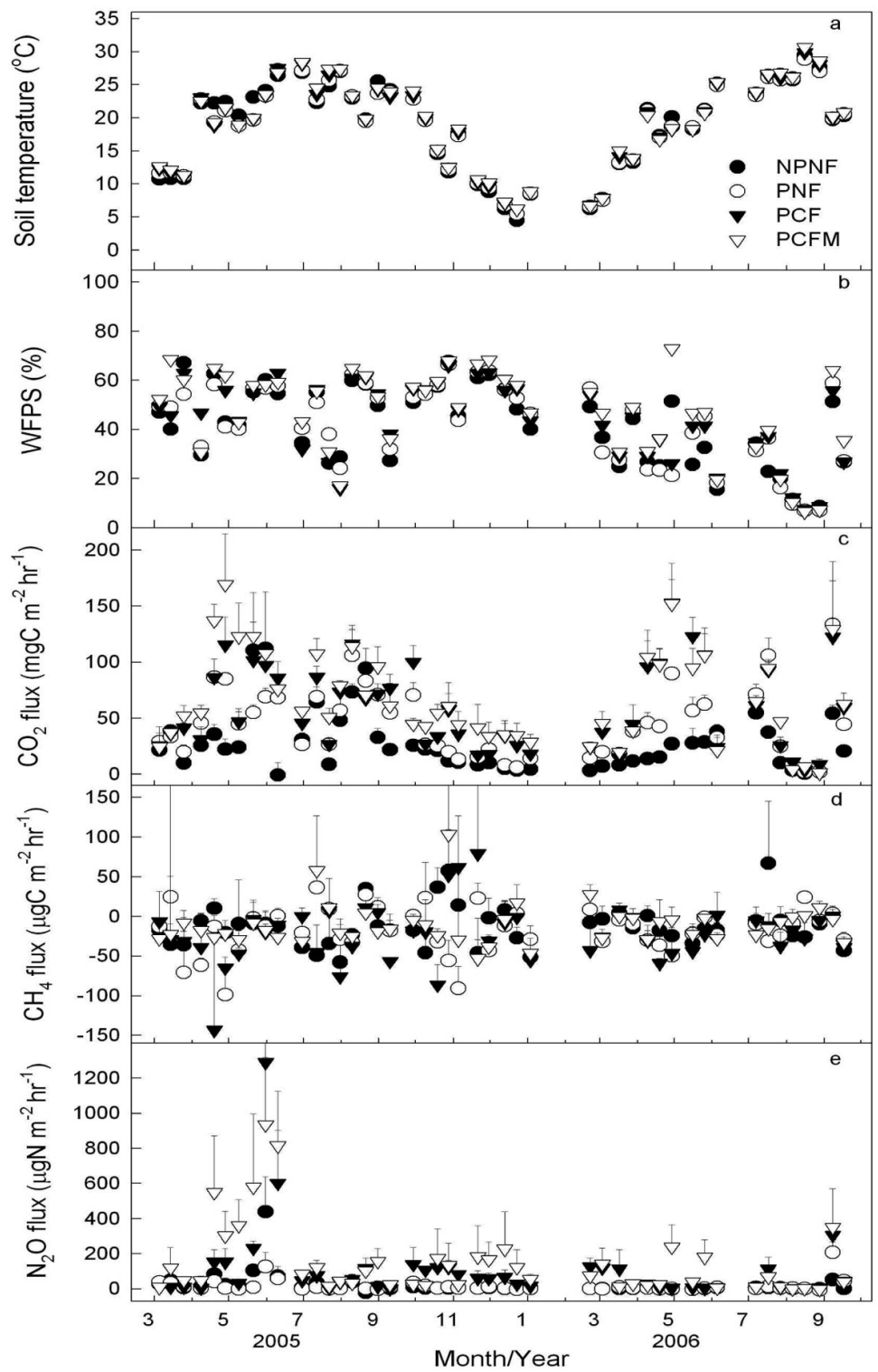

Figure 1. Seasonal pattern of (a) soil temperature, (b) water-filled pore space (WFPS), and fluxes of (c) $\mathrm{CO}_{2}$, (d) $\mathrm{CH}_{4}$ and (e) $\mathrm{N}_{2} \mathrm{O}$ from different treatments at the site CS. Error bars indicate the standard error $(\mathrm{n}=3)$. 
Unlike site CS, site CJ showed the highest $\mathrm{N}_{2} \mathrm{O}$ emissions in the treatments PCF and PCFM following rewetting of soils in early September 2006 after a lasting summer drought (WPFS $<40 \%$ ) over the preceding two months (Figure 2). The pulses of higher $\mathrm{N}_{2} \mathrm{O}$ fluxes were also observed following fertilization and/or rewetting of soils during other periods, especially in the first year. Across the entire study period, the average $\mathrm{N}_{2} \mathrm{O}$ fluxes from different treatments ranged from 16.8 to $146 \mu \mathrm{g} \mathrm{N} \mathrm{m}^{-2} \mathrm{hr}^{-1}$ for site CS (Table 2), and from 26.8 to $287 \mathrm{~g} \mathrm{~N} \mathrm{~m}^{-2} \mathrm{~h}^{-1}$ for site CJ (Table 3). The total $\mathrm{N}_{2} \mathrm{O}$ emission in different treatments ranged from 1.9 to $18.7 \mathrm{~kg} \mathrm{~N} \mathrm{ha}^{-1}$ for site CS (Table 2) and from 3.3 to $33.4 \mathrm{~kg} \mathrm{~N} \mathrm{ha}^{-1}$ for site CJ (Table 3). Although comparable rates of fertilizer $\mathrm{N}$ were applied to soils at both sites, the $\mathrm{N}_{2} \mathrm{O}$ fluxes in the treatments at site $\mathrm{CJ}$ were about 2 times greater than those in the corresponding treatments at site CS. Compared with site CS, site CJ showed relatively high soil temperature and moisture over the entire study period and contained a $60 \%$ higher organic carbon in soils, all of which suggests that soil conditions at site CJ were more favorable for $\mathrm{N}_{2} \mathrm{O}$ production through denitrification and nitrification.

For both sites, the cumulative $\mathrm{N}_{2} \mathrm{O}$ emissions in the unfertilized treatments PNF and NPNF were often not statistically different, indicating that the $\mathrm{N}_{2} \mathrm{O}$ emission in each of these two treatments could be taken as the background emission. If the $\mathrm{N}_{2} \mathrm{O}$ emission in PNF was assumed to be the background emission, the fertilizer-induced $\mathrm{N}_{2} \mathrm{O}$ emission factor (EF) of individual crop phases would vary from 0.2 to $3.9 \%$ for site CS (Table 2) and from 0.8 to $5.1 \%$ for site CJ (Table 3). Linear regression analysis showed that about $58 \%$ of the seasonal variation in the EFs could be explained by the product (interaction) of mean soil temperature and WFPS of individual crop phases (the EFs in PCF and PCFM at site CJ during the pepper growth phase were highly scattered and thus excluded). This indicated that rectification of EF by temperature and soil moisture or precipitation may be an advisable approach for reducing the uncertainties associated with the emission factor- based $\mathrm{N}_{2} \mathrm{O}$ emission estimates (Lu et al., 2006; Mei et al., 2011). On average for the entire study period and the two fertilized treatments PCF and PCFM, the EF was $1.4 \pm 1.1 \%$ (standard deviation of ten observations) for site CS and $3.1 \pm 1.7 \%$ (standard deviation of eight observations) for site CJ. These mean values were within the range of 0.59 to $4.98 \%$ of annual emission factor observed in other vegetable fields in southeastern China (Mei et al., 2011), but were larger than the latest IPCC (2006) default value (1.0\%), implying a potential substantial contribution of vegetable cultivation to national $\mathrm{N}_{2} \mathrm{O}$ emission inventory.

\subsection{Global warming potential}

The overall balance of the net exchange of $\mathrm{CO}_{2}, \mathrm{~N}_{2} \mathrm{O}$ and $\mathrm{CH}_{4}$ constitutes the net GWP of a soil. All the soils under different treatments at sites CS and CJ were net contributors to global warming since the net GWPs were always positive during each of the crop phases (Table 2 and 3). For the whole study period, the net GWPs in different treatments ranged from 1,401 to $1,861 \mathrm{~g} \mathrm{CO}_{2}$ $\mathrm{m}^{-2}$ at site $\mathrm{CS}$ and from 2,537 to 3,596 $\mathrm{g} \mathrm{CO}_{2} \mathrm{~m}^{-2}$ at site CJ. The net GWP in each treatment at site $\mathrm{CJ}$ was about two times as high as that in the corresponding treatments at site CS. The net GWPs from soils at site CS were within the range reported in other studies of agricultural cropping systems under conventional management, while the net GWPs at site CJ were relatively high. It was found that the net GWPs varied from 749 to 1,790 $\mathrm{g} \mathrm{CO}_{2} \mathrm{~m}^{-2}$ for various upland soil cropping systems in central Hokkaido of Japan (Mu et al., 2006) and from 7 to $250 \mathrm{~g} \mathrm{CO}_{2} \mathrm{~m}^{-2}$ for conventional-till continuous corn systems in northeastern Colorado (Mosier et al., 2006). The soil $\mathrm{CH}_{4}$ emission from the different treatments was equivalent to -10 to $-6 \mathrm{~g} \mathrm{CO}_{2} \mathrm{~m}^{-2}$ at site $\mathrm{CS}$ and 0 to $1 \mathrm{~g} \mathrm{CO}_{2} \mathrm{~m}^{-2}$ at site CJ. The contribution of $\mathrm{CH}_{4}$ to the overall net GWP was lower than $1 \%$ for both sites and therefore can be negligible. On the other hand, the relative contribution of net $\mathrm{CO}_{2}$ emission to net GWP ranged from 90 to $95 \%$, and the contribution of $\mathrm{N}_{2} \mathrm{O}$ ranged from 5 to $10 \%$ for the unfertilized treatments NPNF and PNF at both sites. This was well within the range of 84 to $99 \%$ contribution from $\mathrm{CO}_{2}$ emission and 
1 to $16 \%$ from $\mathrm{N}_{2} \mathrm{O}$ to net GWP observed by $\mathrm{Mu}$ et al. (2006) and Shrestha et al. (2009). In contrast, the contribution of $\mathrm{N}_{2} \mathrm{O}$ to net GWP increased to 33 to $47 \%$, while the contribution of $\mathrm{CO}_{2}$ decreased to 53 o $67 \%$ for the fertilized treatments PCF and PCFM. This highlighted the importance of reduction of fertilizer-induced $\mathrm{N}_{2} \mathrm{O}$ emission as well as stabilization and increase of soil carbon storage through suitable management to mitigate the global warming effect of vegetable fields.

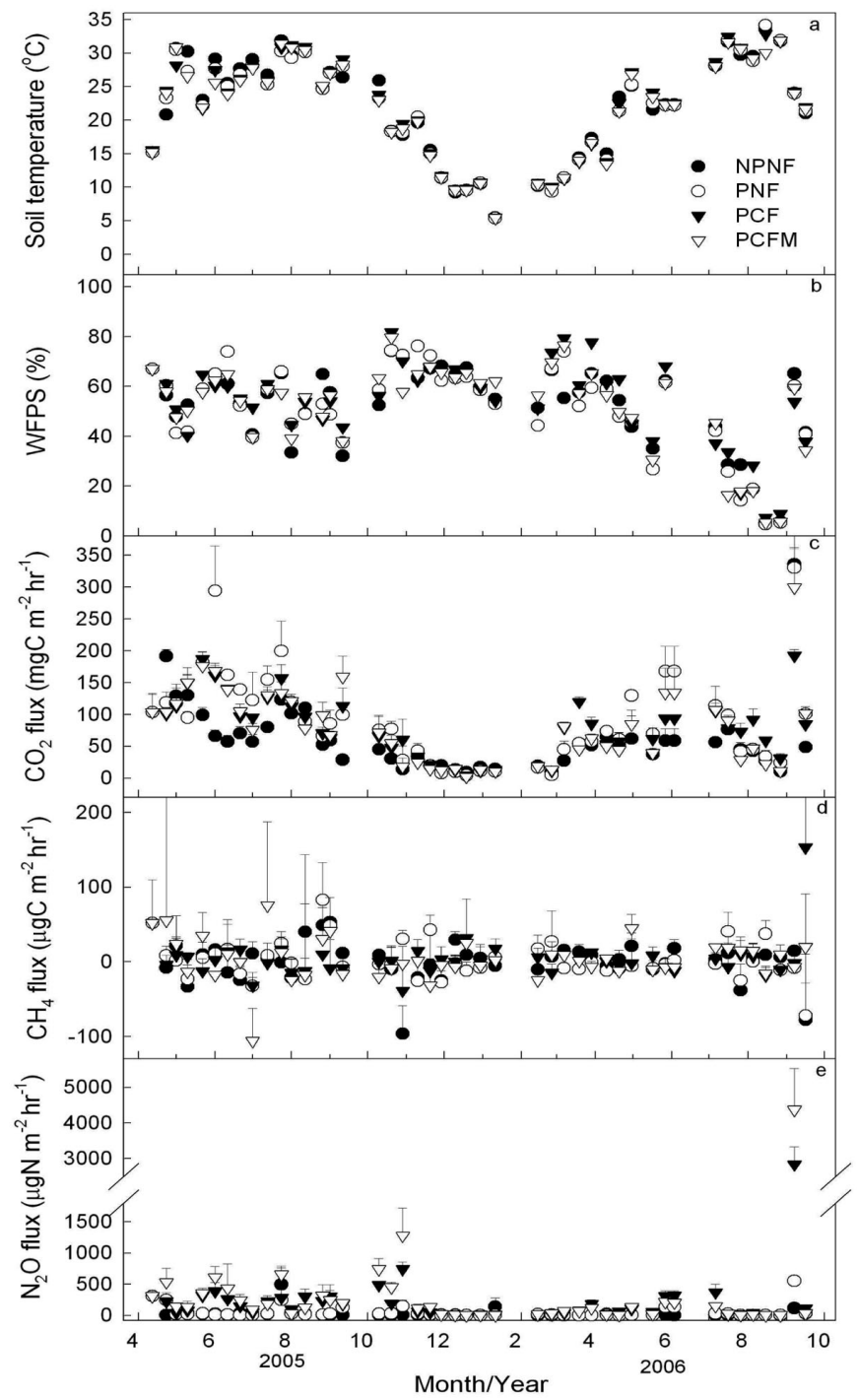

Figure 2. Seasonal pattern of (a) soil temperature, (b) water-filled pore space (WFPS), and fluxes of (c) $\mathrm{CO}_{2}$, (d) $\mathrm{CH}_{4}$ and (e) $\mathrm{N}_{2} \mathrm{O}$ from different treatments at the site CJ. Error bars indicate the standard error $(\mathrm{n}=3)$ 


\section{Conclusion}

Greenhouse gas fluxes from two typical vegetable fields subjected to local conventional management practices showed a large temporal and inter-site variation. Seasonal pattern of intra-site GHG fluxes was mainly influenced by soil temperature and moisture, while the inter-site variation appeared to be regulated also by other soil properties such as texture, organic carbon and $\mathrm{N}$ availability. Intensive fertilization consistently stimulated soil $\mathrm{N}_{2} \mathrm{O}$ emission but imposed a confounding effect on soil respiration with $\mathrm{CO}_{2}$ emission stimulated at one site and suppressed at the other site. All the soils under different vegetable cropping systems were net sources of global warming with net $\mathrm{CO}_{2}$ emission contributing $53 \%$ to $67 \%$ of the overall net GWP from fertilized soils and $\mathrm{N}_{2} \mathrm{O}$ emission contributing the remaining $33 \%$ to $47 \%$. These findings suggest that sustainable management practices are pressingly needed to explore for vegetable farming to satisfy the increasing demand for vegetable supply while to mitigate its global warming effect through reducing fertilizer-induced $\mathrm{N}_{2} \mathrm{O}$ emission as well as increasing carbon sequestration in vegetable fields.

\section{Acknowledgements}

This work was supported by the National Major Science and Technology Projects for Water Pollution Control and Management (2012ZX07104-003), the Fundamental Research Funds for the Central Universities of China (SWU-XDJK2010C045), the National Natural Science Foundation of China (No 41061140515), and the Natural Science Foundation Project of CQ CSTC (2008BB7004).

\section{References}

Adviento-Borbe, M.A.A., Haddix, M.L., Binder, D. L., Walters, D.T. 2007. Soil greenhouse gas fluxes and global warming potential in four high-yielding maize systems. Global Change Biol. 13, 1972-1988.

Chinese Statistical Bureau. 2010. China Statistical Yearbook. Chinese Statistical Bureau, Beijing, China.

Cole, C.V., Duxbury, J., Freney, J., Heinemeyer, O., Minami, K., Mosier, A., Paustian, K., Rosenberg, N., Sampson, N., Sauerbeck, D., Zhao, Q. 1997. Global estimates of potential mitigation of greenhouse gas emissions by agriculture. Nutri. Cycl. Agroecosys. 49, 221-228.

CRGCST (Cooperative Research Group on Chinese Soil Taxonomy, Coordinated by Institute of Soil Science, Chinese Academy of Sciences). 2001. Chinese Soil Taxonomy. Science Press, Beijing, New York. pp 203.

Guo, J.H., Liu, Z.J., Zhang, Y., Shen, J.L., Han, W. X., Zhang, W.F., Christie, P., Goulding, K.W.T., Vitousek, P.M., Zhang, F.S. 2010. Significant acidification in major Chinese croplands. Science. 327, 1008-1010.

Hanson, P. J., Edwards, N.T., Garten, C.T., Andrews, J.A. 2000. Separating root and soil microbial contributions to soil respiration: a review of methods and observations. Biogeochemistry. 48, 115-146.

Hassink, J., Bouwman, L.A., Zwart, K.B., Bloem, J., Brussaard, L. 1993. Relationships between soil texture, physical protection of organic matter, soil biota, and $\mathrm{C}$-mineralization and $\mathrm{N}$-mineralization in grassland soils. Geoderma. 57, 105-128.

He, F.F., Jiang, R.F., Chen, Q., Zhang, F.S., Su, F. 2009. Nitrous oxide emissions from an intensively managed greenhouse vegetable cropping system in Northern China. Environmental Pollution. 157, 1666-1672. 
IPCC. 2007. Climate Change 2007: Synthesis Report. IPCC, Geneva, Switzerland, 104 p.

IPCC. 2006. Guidelines for National Greenhouse Gas Inventories (Volume 4). IPCC National Greenhouse Gas Inventories Programme \& Institute for Global Environmental Strategies, Kanagawa, Japan.

Jia, S.X., Wang, Z.Q., Li, X.P., Sun, Y., Zhang, X.P., Liang, A.Z. 2010. N fertilization affects on soil respiration, microbial biomass and root respiration in Larix gmelinii and Fraxinus mandshurica plantations in China. Plant and Soil. 333, 325-336.

Linn, D.M., Doran, J.W. 1984. Effect of water-filled pore space on carbon dioxide and nitrous oxide production in tilled and no-tilled soils. Soil Sci. Soc. Am. J. 48, 1267-1272.

Liu, M., Ekschmitt, K., Zhang, B., Holzhauer, S.I. J., Li, Z.P., Zhang, T.L., Rauch, S. 2011. Effect of intensive inorganic fertilizer application on microbial properties in a paddy soil of subtropical China. Agricultural Sciences in China. 10, 17581764.

Lu, Y.Y., Huang, Y., Zou, J.W., Zheng, X.H. 2006. An inventory of $\mathrm{N}_{2} \mathrm{O}$ emissions from agriculture in China using precipitation-rectified emission factor and background emission. Chemosphere. $65,1915-1924$.

Maag, M., Vinther, F.P. 1999. Effect of temperature and water on gaseous emissions from soils treated with animal slurry. Soil Sci. Soc. Am. J. 63, 858865 .

Mei, B.L., Zheng, X.H., Xie, B.H., Dong, H.B., Yao, Z.S., Liu, C.Y., Zhou, Z.X., Wang, R., Deng, J., Zhu, J.G. 2011. Characteristics of multipleyear nitrous oxide emissions from conventional vegetable fields in southeastern China. J. Geophys. Res. 116, D12113, doi:10.1029/2010JD015059.
Mosier, A.R., Halvorson, A.D., Reule, C.A., Liu, X.J. 2006. Net global warming potential and Greenhouse Gas intensity in irrigated cropping systems in Northeastern Colorado. J. Environ. Qual. 35, 1584-1598.

Mu, Z.J., Kimura, S.D., Hatano, R. 2006. Estimation of global warming potential from upland cropping systems in central Hokkaido, Japan. Soil Sci. Plant Nutri. 52, 371-377.

Mu, Z.J., Kimura, S.D., Toma, Y., Hatano, R. 2008a. Nitrous oxide fluxes from upland soils in central Hokkaido, Japan. J. Environ. Sci. 20, 1312-1322.

Mu, Z.J., Kimura, S.D., Toma, Y., Hatano, R. 2008b. Evaluation of the soil carbon budget under different upland cropping systems in central Hokkaido, Japan. Soil Sci. Plant Nutri. 54, 650-661.

Rey, A., Pegoraro, E., Tedeschi, V., Deparri, I., Jarvis, P.G., Valentini, R. 2002. Annual variation in soil respiration and its components in a coppice oak forest in Central Italy. Global Change Biol. 8, 851-866.

Robertson, G.P., Paul, E.A., Harwood, R.R. 2000. Greenhouse gases in intensive agriculture: contributions of individual gases to the radiative forcing of the atmosphere. Science. 289, 19221925.

Shrestha, R.K., Lal, R., Penrose, C. 2009. Greenhouse gas emissions and global warming potential of reclaimed forest and grassland soils. J. Environ. Qual. 38, 426-436.

Tian, Y.Q., Liu, J., Wang, X.H., Gao, L.H. 2011. Carbon mineralization in the soils under different cover crops and residue management in an intensive protected vegetable cultivation. Scientia Horticulturae. 127, 198-206.

Wang, H., Dong, Y.H., Li, D.C., Velde, B., An, Q., Guo, Z.X., Zuo, Q.D. 2008. Evolution of soil 
chemical properties in the past 50 years in the Tai Lake region, China. Soil and Till. Res. 100, 54-59.

Zheng, X.H., Han, S.H., Huang, Y., Wang, Y.S., Wang, M.X. 2004. Re-quantifying the emission factors based on field measurements and estimating the direct $\mathrm{N}_{2} \mathrm{O}$ emission from Chinese croplands. Global Biogeochem. Cycles. 1 GB2018, doi:10.1029/2003GB002167.
Zhu, T. B., Zhang, J. B., Cai, Z. C. 2011. The contribution of nitrogen transformation processes to total $\mathrm{N}_{2} \mathrm{O}$ emissions from soils used for intensive vegetable cultivation. Plant and Soil. 343, 313-327. 